\title{
Research Article \\ Study of Systems Error Compensation Methods Based on Molecular-Electronic Transducers of Motion Parameters
}

\author{
Dmitry L. Zaitsev (D), Vadim M. Agafonov (D, and Iliya A. Evseev \\ Moscow Institute of Physics and Technology, Moscow 117303, Russia \\ Correspondence should be addressed to Dmitry L. Zaitsev; zaitcev.dl@mipt.ru
}

Received 19 October 2017; Accepted 17 May 2018; Published 19 June 2018

Academic Editor: Jesus Corres

Copyright (C) 2018 Dmitry L. Zaitsev et al. This is an open access article distributed under the Creative Commons Attribution License, which permits unrestricted use, distribution, and reproduction in any medium, provided the original work is properly cited.

\begin{abstract}
The main objective of the paper is to study the system errors of azimuth determination in the dynamic scheme of north finding on the base of the molecular-electronic sensitive angular motion sensor. Introduced theoretical and experimental study of some error compensation methods. Investigated the most significant system inaccuracies of azimuth determination depended on MET sensor g-sensitivity factor and the occurrence of rotation uneven in the system and as a result of tiny angular accelerations which appeared. Methods and algorithms of error reduce are experimentally verified.
\end{abstract}

\section{Introduction}

The crucial task of the modern stage of the development of technology is the problem of the exact binding of various measuring and navigation systems to the true north heading. The importance of high-precision determination of true azimuth is emphasized by a number of practical applications in registration, navigation, targeting problem solving and object direction determination, high-precision orientation of communication systems, geodesy, and so on.

The traditional way to determine azimuth is to measure the direction with the help of a magnetic compass, considering (or not) the terrain magnetic declination. Although this method has been known for a long time, noncorrespondence of the magnetic and geographic poles, as well as the presence of magnetic anomalies or magnetized objects nearby, makes the compass usage inaccurate for the solution of the specified tasks of geographical north finding [1].

The solution of the problem of positioning and determining the motion direction in a number of cases can be achieved with the help of modern satellite systems, such as GPS/GLONASS [2]. However, these methods, being based on external signals, are by definition heteronomous, which can be critical for some applications, such as for navigational tasks of underwater vehicles or for positioning in closed or underground facilities. Besides, to achieve high accuracy in direction determination, it is necessary to conduct measurements at several points, which are remote from each other, as well as to use high-precision signal receivers, providing high accuracy in the determination of the coordinates of each point.

Modern methods of high-precision orientation determination include a wide usage of various gyrocompass devices [3]. However, the use of high-precision gyrocompasses with the accuracy of determining the azimuth direction better than $0.5^{\circ}$ is substantially limited by their cost, consumption, and mass-size parameters.

An alternative to the established trend of various gyroscopic systems development to solve the task of direction determination is an autonomous method of object azimuth finding based on finding of Earth angular velocity vector by measuring the Coriolis forces with a linear accelerometer rotating around an axis parallel to the sensitivity axis of that accelerometer [4]. At the same time, the accuracy of this method is not large due to the smallness of the Coriolis forces in comparison to the typical external noise vibrational background.

From the point of view of technical implementation and high accuracy attainability in finding the geographic north heading, a better option is a well-known method based on 
the direct finding of Earth angular velocity vector by means of an angular accelerometer rotating about the axis orthogonal to the sensitivity axis of that accelerometer. The principle of the method is the modulation of the Earth rotation rate signal by forced mechanical rotation of the angular accelerometer. In case the rotation axis is not parallel to $\overrightarrow{\Omega_{e}}$, the projection $\omega_{e}$ of the Earth angular velocity vector on the axis of sensitivity of the angular accelerometer, and hence on its output signal, alternates with the accelerometer rotation, which makes it possible to determine the position of the sensitivity axis lying in the same plane as the Earth angular velocity vector $\overrightarrow{\Omega_{e}}$ and consequently the geographical north heading $[5,6]$. The described method could be quite effective granted that there is a sufficiently sensitive angular motion sensor. At the same time, none of the angular motion sensors, including microelectromechanical and fiber-optic, available until quite recently, give the high accuracy necessary in their usage. Nevertheless, the solution of the task of high-precision finding of the azimuth direction with the help of this method is achievable with the use of a more sensitive and accurate angular sensor. Molecularelectronic technology-based angular sensor [7-10] can be used in this case. The described solution is implemented in the patent [11]. Besides, the patent [5] on the inertial determinant of the geographical north heading is very similar to it. The solution is a nongyroscopic inertial system that allows to determine the geographic north azimuth. The system operates with the use of a liquid angular motion sensor mounted on a rotating platform. The sensor is placed on a platform capable of rotating at a certain constant angular velocity so that its sensitivity axis to the angular motion is orthogonal to the platform rate of rotation vector.

\section{Description of the Assembly and Measurement Scheme}

In this paper, an autonomous system of finding the azimuth by a dynamic method based on a molecular-electronic angular velocity sensor will be studied in detail. The basic scheme of the assembly operation is shown in detail in [12]. Zaitsev et al. [13] provide the experimental results of the scheme study from [12]. In the present work, the study of the proposed system based on molecular-electronic sensors is continued. According to what has been said, the description of the experimental assembly is schematically shown in Figure 1.

The rotating platform 1 is equipped with a molecularelectronic angular motion sensor 2 (SA: sensitive axis) and an inclinometer 3 detecting the deviation of the site from the horizontal position. The platform is driven by motors 4 . At the site, next to the rotating platform, a magnetic encoder 5 is installed. The encoder registers the position of the platform in time with respect to the start position. Signals from the sensors are registered in the data acquisition unit 6 , processed in block 7, and visualized on the computer real time, which allows to control sensor signals clearly, including nonuniformity of the platform rotation. Figure 2 shows a user window where the platform rotation is controlled and the signals from the sensors are monitored real time. The device

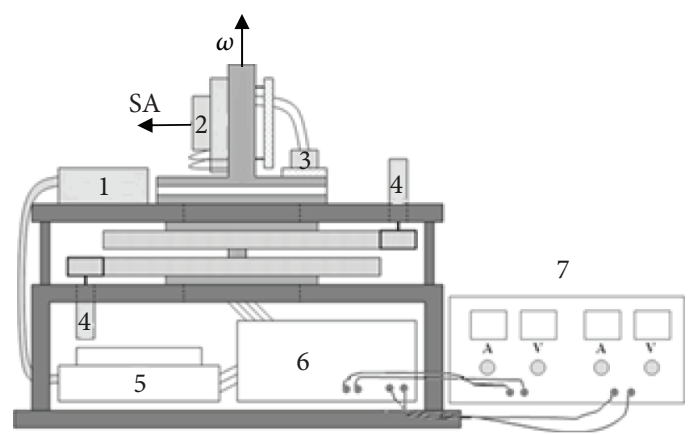

FIgURE 1: Schematic design of the device to measure the geographical north heading.

is powered by the power supply 8 . At that, 4 signals are visualized, as shown in Figure 2. White shows the signal of the molecular-electronic sensor, by which the azimuth is determined, red shows the signal of the angle sensor, which records the change in inclination of the platform during rotation, green shows the platform angle sensor (magnetic encoder), and blue shows the derivative of the encoder signal, which demonstrates that the platform with sensors rotates evenly. Figure 3 shows the general view of the experimental assembly.

\section{Problem Statement}

The accuracy of the azimuth determination by the developed device and the measurement method generally depends on a number of factors, in particular, on the orthogonality degree of the platform rotation axis of and the angular motion sensor sensitivity axis and rotation axis beatings, which change the slope of the angular motion sensor sensitivity axis in relation to the acceleration of gravity, which will also be seen in the appearance of a periodic signal at the sensor output in case of corresponding sensitivity to linear accelerations [14]. The measurement accuracy in accordance with [12] is influenced directly by the primary sensor self-noise. Besides, temperature drift $[15,16]$ and special aspects of the output to the mode of the molecular-electronic sensor must be taken into account $[17,18]$.

In this paper, we will consider two factors that determine the special aspects of the dynamic method of azimuth finding with the help of the latest molecular-electronic angular motion sensors.

The signal of the molecular-electronic angular motion sensor after being brought to the rotation in accordance with the dynamic method algorithm and calculation of the Fourier transform at the platform rotation frequency is as follows:

$$
\begin{aligned}
U_{\text {sens }}(\tilde{\omega})= & \Omega * \exp (i \delta)+U_{\text {gsens }}(\tilde{\omega}) \\
& +U_{\text {enc }}(\tilde{\omega}) * \sin \alpha+U_{\text {noise }}(\tilde{\omega}),
\end{aligned}
$$

where $\Omega$ is the projection of the Earth angular velocity on the rotation plane of the molecular-electronic sensor sensitivity axis, $\delta$ is the phase of the output useful signal, $\tilde{\omega}$ is the average speed of the platform rotation, $U_{\text {gsens }}$ is the parasitic sensor signal, caused by the rotation axis beating, which contribute 


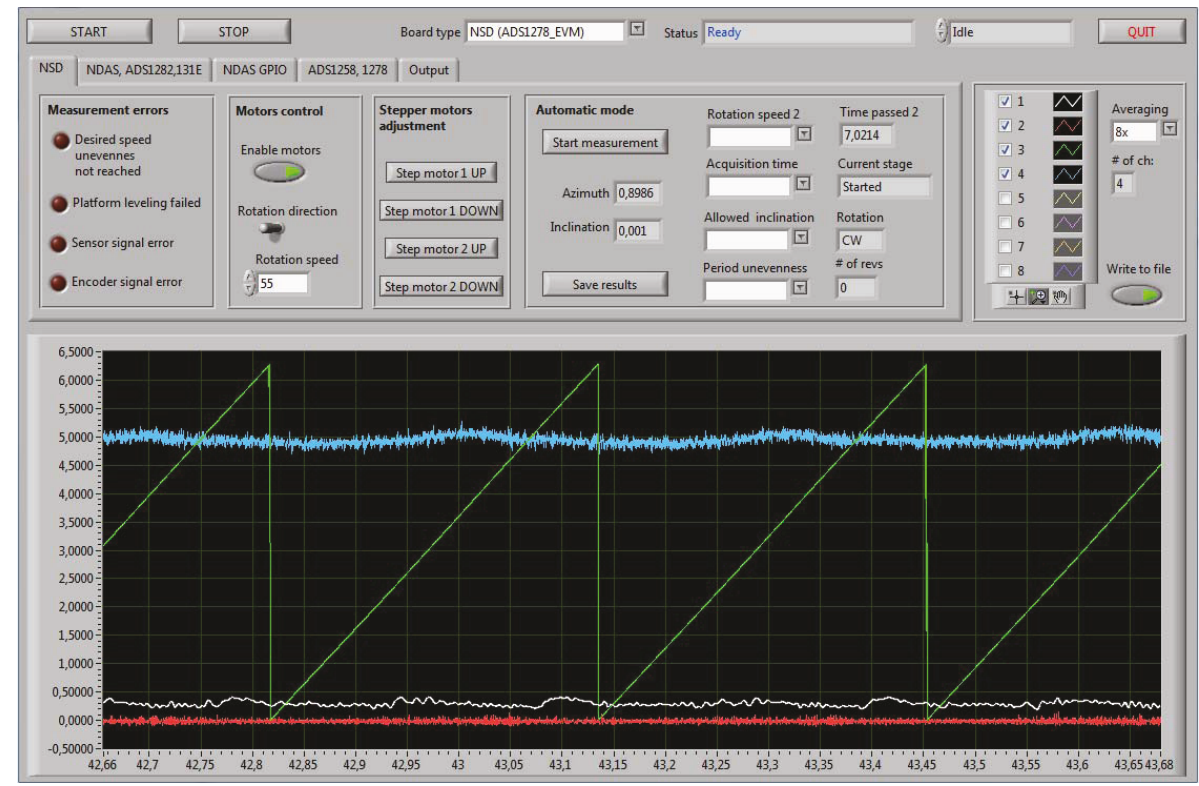

FIGURE. 2: User window and sensor signals at the platform rotation real time. White is the signal of the molecular-electronic sensor, red is the signal of the inclinometer, green is the signal of the magnetic encoder, and blue is the differential of the encoder signal.

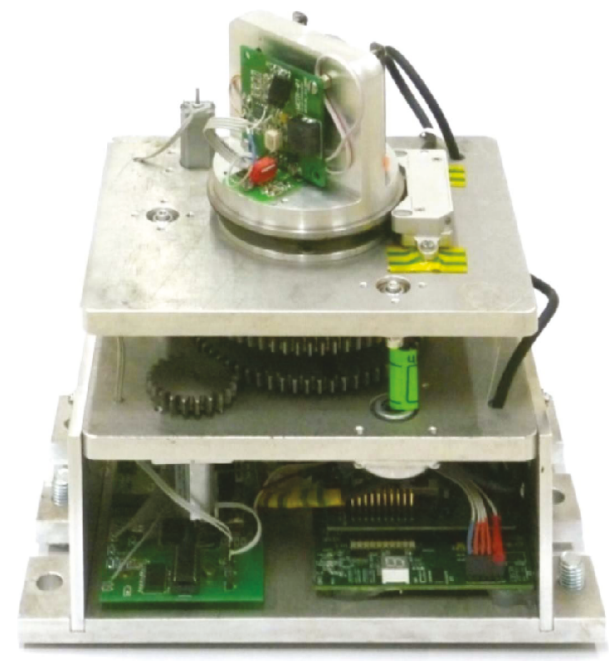

Figure 3: General view of the device for the geographical north finding.

into the system signal due to the sensitivity to linear acceleration and the corresponding changes in the gravity vector projection on the sensitivity axis of the device, $U_{\text {enc }}$ is the encoder signal measuring the platform rotational speed over time, $\alpha$ is the deviation angle of the molecular-electronic sensor sensitivity axis from the perpendicular to the platform angular velocity vector (the angle of nonorthogonality), and $U_{\text {noise }}$ is the self-noise of an angular sensor, considered in detail in $[19,20]$.

The first term represents a useful signal. The second term represents errors due to the influence of the linear acceleration (gravity). The third term is the parasitic signal conditioned by nonuniformity of the platform rotation. The fourth term describes errors associated with the molecularelectronic sensor self-noise. The fourth term was studied in detail in [12]. It was shown that the self-noise of a molecular-electronic sensor was affecting the azimuth error, and numerical and experimental simulations of such effect were carried out. But this kind of error cannot be reduced by the system compensation methods, it can only be reduced by increasing the measurement time, and this error is completely determined by the physical mechanisms of MET sensor's self-noise. In [12], it was demonstrated that, in case of absence of other types of system errors, the azimuth error generated by the sensor's self-noise could be close to $0.1^{\circ}$ at the measurement time of $200 \mathrm{sec}$.

The present study and the second and third terms from (1) will be considered in detail; the nature of the effects will be studied, and the mechanisms for compensating these errors will be proposed.

\section{Theoretical Description of Error Compensation Methods Associated with Linear Acceleration}

One of the reasons for the error in north finding by the proposed above dynamic scheme is the effect of linear accelerations, the second term of (1), on the device sensitive element. Huang et al. [10] offer the solution of the task to eliminate the error in determining the north heading, which is done by exact orientation of the platform rotation axis along the local vertical. In this case, rotation does not change the sensor orientation to gravity. Nevertheless, this solution cannot always be implemented technically with the necessary accuracy.

Now we determine how the errors related to the change in the sensor position to gravity can be corrected if the platform rotation axis is not vertical in a small range of angles. To do this, consider a sensor rotating relative to one of 
the axes located in the plane of the toroidal channel of its body (Figure 4). Introduce a coordinate system which $z$ axis is directed along the platform rotation axis, while $x$ axis is oriented perpendicular to the toroidal plane, that is, along the sensitivity axis of the angular motion sensor. The $y$-axis complements the coordinate system to the right three. Introduce the following notation: $\alpha$ is the inclination angle of the rotation axis relative to the vertical and $\beta$ is the angle between the plane OXZ and the plane containing the $z$-axis and the gravity vector $\vec{g}$. Then the projections of the gravity vector on the axis of the chosen coordinate system will be as follows:

$$
\begin{aligned}
& g_{x}=g * \sin \alpha \cos \beta, \\
& g_{y}=g * \sin \alpha \sin \beta, \\
& g_{z}=g * \cos \alpha .
\end{aligned}
$$

Let, in addition, $\gamma$ be the angle between $\mathrm{OZ}$ and the direction of the local meridian, and $\varphi$ is the counterclockwise angle between the planes $\mathrm{OXZ}$ and the plane containing the angular velocity vector of the Earth and the OZ axis. Then the projection of the angular velocity of the Earth's rotation $\vec{\Omega}$ on the sensitivity axis of the sensor will be:

$$
\Omega_{x}=\Omega * \sin \gamma \sin \phi .
$$

If the liquid in the toroidal channel of the angular motion sensor was strictly homogeneous, then the moment of inertia forces creating the liquid circulation in the channel would be $M=m R^{2} \dot{\Omega}_{x}$. In reality, the liquid density is not strictly constant but particularly depends on a change in its concentration or the temperature gradients. Without making any a priori assumptions about the nature of the density distribution, the moment of forces driving the liquid in the toroidal channel within linear response can be written as follows:

$$
M=m R^{2} \dot{\Omega}_{x}+A_{y} g_{y}+A_{z} g_{z}
$$

The coefficients $A_{z}$ and $A_{y}$ consider the inhomogeneity of the distribution of the fluid density in the toroid channel and related linear acceleration sensor sensitivity. In this calculation, the coefficients should be considered as a priori unknown and to be determined based on the experimental data.

Suppose the platform comes into rotation counterclockwise with angular velocity $\omega$. Then,

$$
\begin{aligned}
& \beta=\beta_{0}+\omega t, \\
& \phi=\phi_{0}+\omega t .
\end{aligned}
$$

Here $\beta_{0}$ and $\phi_{0}$ are the values of the corresponding angles at the initial moment of time $t=0$. Substitute (2), (3), and (5) into (4) and, by preserving only the terms that depend on time, getting

$$
\begin{aligned}
M= & -m R^{2} \Omega \omega \sin \gamma \sin \left(\omega t+\phi_{0}\right) \\
& +A_{y} g \sin \alpha \sin \left(\omega t+\beta_{0}\right)
\end{aligned}
$$

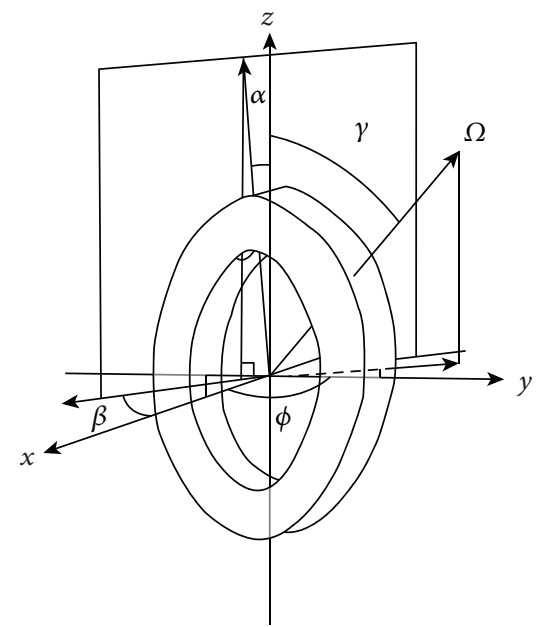

Figure 4: Schematic view of the molecular-electronic sensor of angular motions.

Denote the complex coefficient of the transformation of the moment of forces $M$ into the output signal of the sensor by $W(\omega)$. Using (6), find the following for the output signal when rotating counterclockwise:

$$
U_{\mathrm{ccw}}(\omega)=\frac{W(\omega)}{2 i} *\left(-f_{E} \exp \left(i \phi_{0}\right)+f_{G} \exp \left(i \beta_{0}\right)\right)
$$

Here, $f_{E}=m R^{2} \Omega \omega \sin \gamma ; f_{G}=A_{y} g \sin \alpha$. follows:

Similarly, for the rotation in the opposite direction, it is as

$$
U_{\mathrm{cW}}(\omega)=\frac{W(\omega)}{2 i} *\left(-f_{E} \exp \left(-i \phi_{0}\right)-f_{G} \exp \left(-i \beta_{0}\right)\right)
$$

Calculate the ratio (7) to (8) and find

$$
\begin{aligned}
\frac{U_{\mathrm{ccw}}(\omega)}{U_{\mathrm{cw}}(\omega)} & \equiv R * \exp (i * \varepsilon) \\
& =\frac{\exp \left(i \phi_{0}\right)-G \exp \left(i \beta_{0}\right)}{\exp \left(-i \phi_{0}\right)+G \exp \left(-i \beta_{0}\right)} .
\end{aligned}
$$

Here, $G=f_{G} / f_{E}$, and $R$ and $\varepsilon$ are the amplitude and phase of polar coordinate system for relationship, respectively, the amplitude and phase relationship $U_{c c w}(\omega) / U_{c w}(\omega)$. Since $U_{\mathrm{ccw}}(\omega)$ and $U_{\mathrm{cw}}(\omega)$ are experimentally determined output signals of the angular motion sensor. Accordingly, $R$ and $\varepsilon$ should be regarded as quantities determined from the experiment. Solving (9), relative to $G$, find

$$
\begin{aligned}
G= & \frac{\left(1-R^{2}\right) \cos \left(\phi_{0}-\beta_{0}\right)}{1+R^{2}+2 R \cos \left(\varepsilon-2 \beta_{0}\right)}+i \\
& * \frac{\left(1+R^{2}\right) \sin \left(\phi_{0}-\beta_{0}\right)-2 R \sin \left(\varepsilon-\phi_{0}-\beta_{0}\right)}{1+R^{2}+2 R \cos \left(\varepsilon-2 \beta_{0}\right)} .
\end{aligned}
$$

Take into account that $G$ is a real value, and get 


$$
\begin{gathered}
\left(1+R^{2}\right) \sin \left(\phi_{0}-\beta_{0}\right) \\
-2 R \sin \left(\varepsilon-\phi_{0}-\beta_{0}\right)=0, \\
G=\frac{\left(1-R^{2}\right) \cos \left(\phi_{0}-\beta_{0}\right)}{1+R^{2}+2 R \cos \left(\varepsilon-2 \beta_{0}\right)} .
\end{gathered}
$$

Thus, if the direction of the rotation axis inclination (the angle $\beta_{0}$ in our description) was known, the error associated with the deviation of the platform rotation axis from the vertical could be eliminated and the direction of the Earth angular velocity relative to the initial position of the platform could be determined by finding $\phi_{0}$ from (11).

In turn, to find the angle $\beta_{0}$, two methods could be proposed:

(1) The method of platform rotation at two different angular velocities: suppose that two series of experiments are consistently carried out. These experiments consist of successive clockwise and counterclockwise rotation of the platform, with the described above two different rotational speeds $\omega$ and $S \omega$. Then, in the second test, as follows from the definition of the parameter $G$, its value for the second series of experiments will be $S$ times less.

Denote the amplitude and relationship phase of the of the sensor signals when rotating counterclockwise and clockwise by $R_{s}$ and $\varepsilon_{s}$ in the second of the experiments, with rotation at angular velocity $S \omega$.

Then, in addition to (9), get

$$
\begin{aligned}
R_{s} * \exp \left(i * \varepsilon_{S}\right) & =\frac{\exp \left(i \phi_{0}\right)-(G / S) \exp \left(i \beta_{0}\right)}{\exp \left(-i \phi_{0}\right)+(G / S) \exp \left(-i \beta_{0}\right)}, \\
G^{2} & =-\frac{D-S D_{S}}{D-D_{S} / S}, \\
D & =-\frac{1-R^{2}}{1+R^{2}}, \\
D_{S} & =-\frac{1-R_{S}^{2}}{1+R_{S}^{2}}, \\
\cos \gamma & =\frac{\left(S^{2}-1\right) D D_{S}}{2 S \sqrt{-\left(D-S D_{S}\right)\left(D-D_{S} / S\right)}},
\end{aligned}
$$

$\sin \left(\varepsilon-2 \phi_{0}\right)=\frac{G^{2} S \sin 2 \gamma}{R D_{S}\left(S^{2}-1\right)(D+1)}$.

The last expression with allowance for (14) allows us to find the required angle $\phi_{0}$.

(2) Simultaneously with the angular motion sensor, a gravity-sensitive sensor (sensitive to the inclination angle), for example, an accelerometer, is placed on the platform: let, for simplicity, the accelerometer sensitivity axis is directed along the OY axis, that is, it lies in the toroid plane. Then the accelerometer

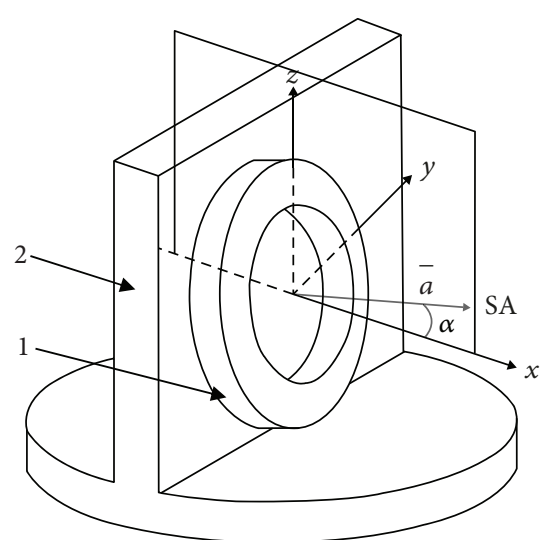

FIgURE 5: Molecular-electronic sensor of angular motion on a rotating platform. 1: mobile platform; 2: molecular-electronic sensor.

output signals at rotation counterclockwise or clockwise measured in the experiment are given by the following expressions:

$$
U_{A}^{+}=\frac{W_{A}(\omega)}{2 i} g \sin \alpha \exp \left(i \beta_{0}\right) .
$$

For the output signals ratio:

$$
\begin{aligned}
\frac{U_{A}^{+}}{U_{A}^{-}} & \equiv \exp (i \delta)=-\exp \left(2 i \beta_{0}\right), \\
U_{A}^{-} & =\frac{W_{A}(\omega)}{2 i} g \sin \alpha \exp \left(-i \beta_{0}\right) .
\end{aligned}
$$

From here,

$$
\beta_{0}=\frac{\delta}{2} \pm \frac{\pi}{2}
$$

which makes it possible to determine the unknown angle.

$\beta_{0}$ based on the experimentally determined value $\delta$, the joint solution of (11) and (17) allows to find, in particular, $\phi_{0}$, that is, the geographical north heading, eliminating the errors associated with the influence of the gravity field on the output signal of the angular motion sensor. It should be noted that the choice between the signs "+" and "-" according to (11) does not affect the result.

\section{Compensation of Errors Associated with the Projection of the Unevenness of the Platform Rotation}

Consider (1) once again, namely, the third term of the formula describing the appearance of an additional parasitic signal, caused not by the orthogonality of the sensitivity axis of the angular motion sensor and the platform rotation axis.

The axes nonorthogonality lead to the fact that, apart from the actual angular velocity of the Earth rotation, the signal received from the angular motion sensor has a projection 


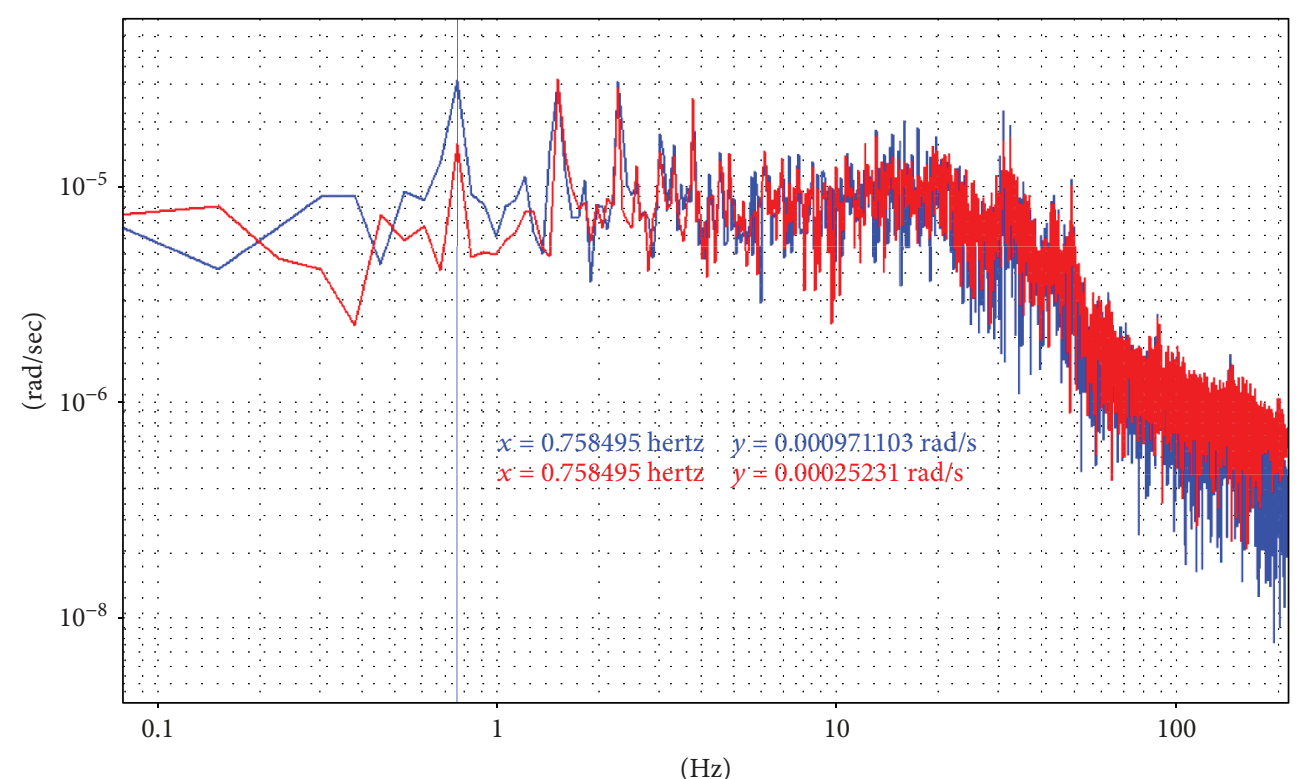

FIgURE 6: Amplitudes of the signal spectra of a molecular-electronic sensor reduced to the dimension rad/s. The blue and red curves show different angles of the sensitivity axis of the molecular-electronic sensor to the platform rotation.

to the sensor sensitivity axis, in general, the nonconstant rotation speed of the platform. Considering the fact that the unevenness of the platform rotation, as a rule, has a periodicity corresponding to the rotation period, that parasitic signal, caused by the unevenness of rotation, is at the same frequency as the measured Earth rotation speed modulated by the platform rotation. Such parasitic signal cannot be eliminated by frequency filtering and by an increase in the averaging signal time. As a result, the accuracy of north finding is significantly reduced.

Consider such situation in more detail. Figure 5 shows the angular motion sensor, marked as 1, attached to the movable platform 2. A coordinate system is introduced, the vector $\vec{a}$ is directed along the sensitivity axis of the angular motion sensor, the $z$-axis is directed along the platform rotation axis, and the $x$-axis is perpendicular to the platform rotation axis so that the plane OXZ contains the vector $\vec{a}$. The $y$-axis complements the coordinate system to the right three. The following notations are introduced: $\alpha$ is the angle between vector $\vec{a}$ and the $x$-axis.

As observed in practice, the fact that the rotation of the platform can be uneven should be considered. Consider how much the nonorthogonality of the position of the sensor sensitivity axis to the platform rotation axis affects the errors in the device readings. If the sensitivity axis is located at some angle to the platform rotation axis different from 90 degrees, then the molecular-electronic sensor, in addition to the Earth rotation, feels the unevenness of the platform rotation, which significantly affects the accuracy of north finding.

To study the effect of the described mechanism on the error in North finding, the data from a magnetic encoder is used. The encoder can measure the platform rotation speed in real time. Let us recall that the magnetic encoder is installed next to the rotating platform (product 5 in Figure 1). With the help of such sensor, the unevenness of the rotation is measured and the received signal is used to adjust the system and process the data further on.

In order to reduce the influence of the parasitic signal on the readings of the molecular-electronic sensor, it is necessary to bring the angular motion sensor into a position where the SA (axis of sensitivity of a sensor) is perpendicular to the platform rotation axis or to subtract the additive from the measured signal due to the discussed effect.

At the controller command, the platform rotation is defined. It is characterized by a strong unevenness (e.g., the platform evenly increases its rotational speed with some constant angular acceleration). In that case, the third term in (1) must be predominant. After calculating the Fourier transform, by the ratio of the signal of the molecular-electronic sensor and the platform speed variation signal from the encoder at a frequency $\tilde{\omega}$ corresponding to some preselected operation frequency of the platform rotation (approximately average value between the maximum and minimum rotation speed), the position of the sensor sensitivity axis to the angular velocity vector of the platform rotation is adjusted by successive tilting of the angular motion sensor in the OXZ plane, minimizing the ratio of the signals $U_{\mathrm{MET}}(\tilde{\omega}) / U_{\text {enc }}(\tilde{\omega})$.

Figure 6 shows the amplitudes of the signal spectra of the molecular-electronic sensor reduced to the dimension $\mathrm{rad} / \mathrm{s}$ at different positions of the sensitivity axis relative to the platform rotation axis. The red curve shows the sensitivity axis deflected from the position of orthogonality by a small angle. The blue curve shows the sensitivity axis deflected by a larger angle. Figure 7 shows the amplitudes of the signal spectra of the magnetic encoder measuring the platform rotational speeds in the corresponding experiments. Rotation is assumed to be uniform in the first approximation.

From the analysis of Figures 6 and 7, it is seen that at the correction of the position of the sensitivity axis of the angular motion sensor, the amplitude of the molecular-electronic 


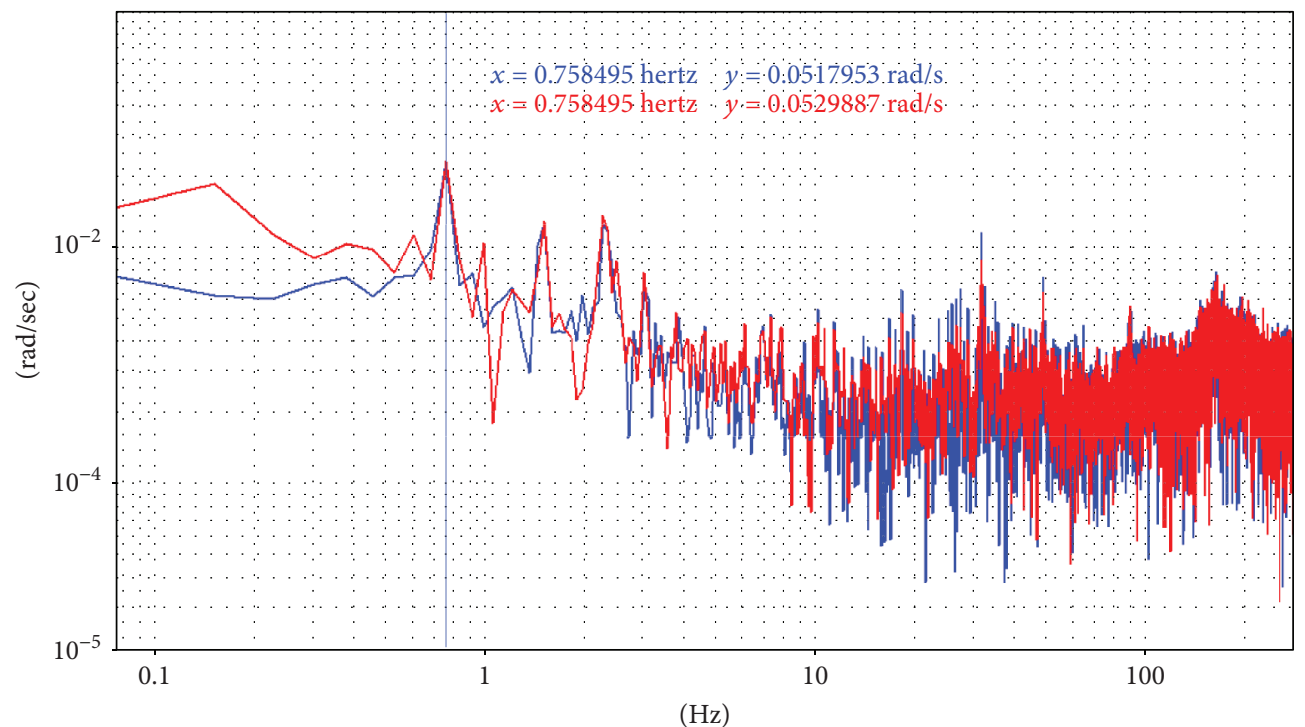

FIgURE 7: Amplitudes of the signal spectra of the platform rotation speed sensor (encoder).

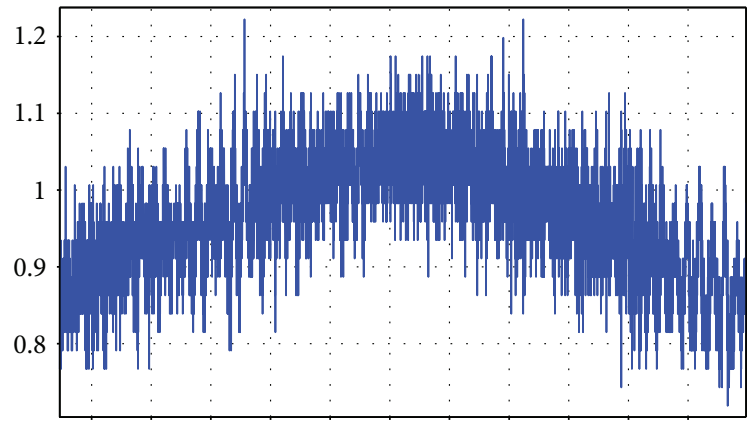

$\begin{array}{lllllllllll}465 & 470 & 475 & 480 & 485 & 490 & 495 & 500 & 505 & 510 & 515\end{array}$

Figure 8: Profile of the change in speed with obviously uneven rotation (the ordinate axis shows $\mathrm{rad} / \mathrm{sec}$ and the abscissa axis shows time in seconds).

sensor signal at the platform rotation frequency decreases. Based on the derivative of the encoder signal, the introduced earlier and denoted in Figure 5 angle $\alpha$ is found from the formula $\tan \alpha=U_{\mathrm{MET}}(\tilde{\omega}) / U_{\mathrm{enc}}(\tilde{\omega})$. Hence, the ratio of the angular motion sensor signal to the derivative of the encoder signal allows to correct the position of the sensor sensitivity axis to the vector of the angular velocity of the platform rotation.

In addition, the determination of the angle of inclination allows to take into account the parasitic signal during further data processing. To do this, the platform is brought into deliberately uneven rotation straight before the measurements (Figure 8 , the velocity profile according to the readings of the encoder), and the coefficient $k$ is measured as the ratio of the signal spectra of the molecular-electronic angular motion sensor (Figure 9, blue curve) to the signal spectrum of the encoder signal derivative (Figure 9, red curve). Thus, a kind of calibration of the signal of the molecular-electronic sensor is performed according to the known signal of the obviously uneven rotation recorded by the angular encoder near the platform rotational speed $\tilde{\omega}$,

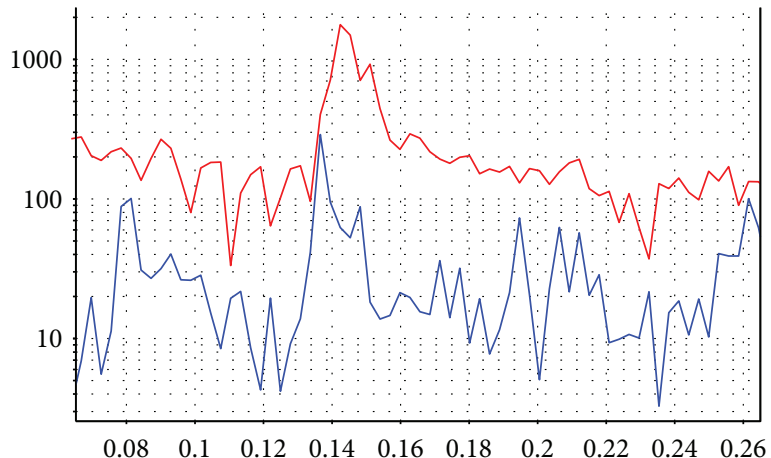

FIGURE 9: Signal spectra of a molecular-electronic sensor and a signal of the encoder's rotational speed with obviously uneven rotation. (The abscissa axis shows frequency in $\mathrm{Hz}$ and the ordinate axis shows relative units).

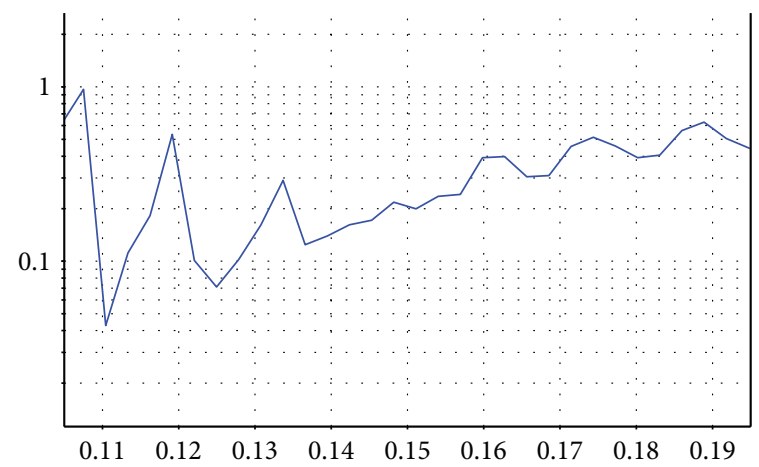

FIGURE 10: Calibration of the molecular-electronic sensor with an uneven rotation signal near the rotation frequency, the sensitivity $k$ of the sensor MEP in this position to the unevenness of rotation in $\mathrm{V} /(\mathrm{rad} / \mathrm{sec})$. (The abscissa axis shows the frequency in $\mathrm{Hz}$ and the ordinate axis shows $\mathrm{V} /(\mathrm{rad} / \mathrm{sec}))$. 


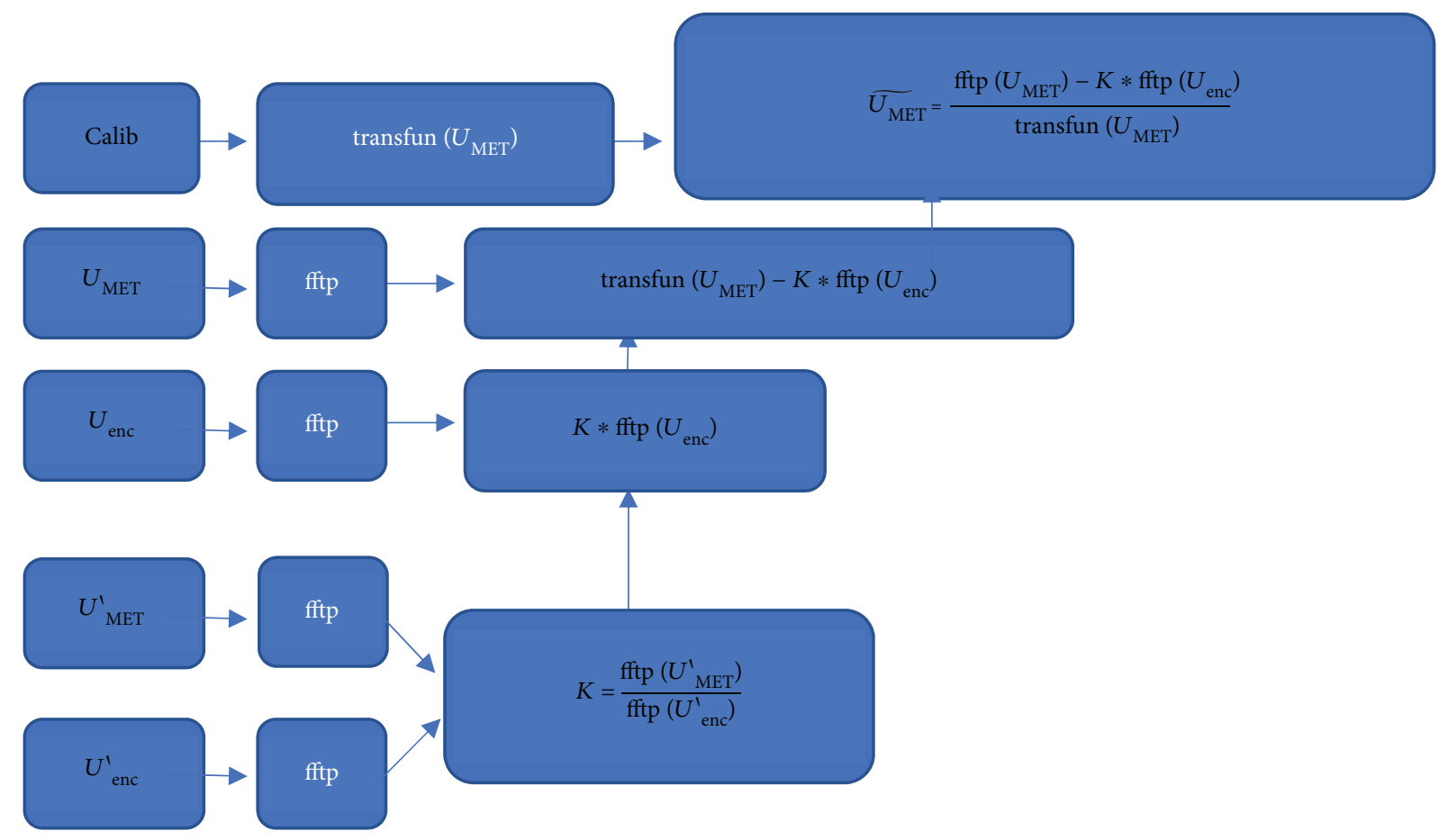

FIGURE 11: The algorithm for error compensation associated with uneven platform rotation.

provided that a strong uneven rotation signal of the platform rotation is predominant in the signal of the molecularelectronic sensor. Figure 10 shows the calibration of the molecular-electronic sensor by a signal of uneven rotation.

Based on the assumption that there is a parasitic signal associated with the unevenness of the platform rotation in the signal of the molecular-electronic sensor, the following formula can be used

$$
U_{\mathrm{MET} \text { corr }}(\tilde{\omega})=U_{\mathrm{MET}}(\tilde{\omega})-U_{\mathrm{enc}}(\tilde{\omega}) * k \text {, }
$$

where $\tilde{\omega}$ is the average rotational speed of the platform with uneven rotation, $U_{\mathrm{enc}}(\tilde{\omega})$ is the encoder signal at the platform rotation frequency, $k$ is the calibration coefficient of the sensitivity to the uneven extra-axial rotation, and $U_{\mathrm{MET}}$ $(\tilde{\omega})$ is the signal of the molecular-electronic sensor at the platform rotation frequency.

Equation (19) describes the correction of the signal of a molecular-electronic sensor with allowance for the unevenness of rotation. Further, by dividing by the transfer function of the molecular-electronic sensor, obtain the MET signal with the account of the uneven motion of the platform.

Schematically, the operation of the algorithm can be represented in the following block diagram in Figure 11.

The following symbols are introduced in the diagram: $\mathrm{C}$ alib is the mechanical calibration of the sensor, transfun is the calibration curve interpolation with the missing number of points, $U_{\mathrm{MET}}$ is the record of the conditionally "uneven" signal of the MET sensor, $U_{\text {enc }}$ is the record of the conditionally "even" encoder signal, $U^{\prime}{ }_{\text {MET }}$ is the record of the obviously uneven signal of the MET sensor, $U^{\prime}{ }_{\text {enc }}$ is the record of obviously uneven signal of the encoder, and $\mathrm{ff}$

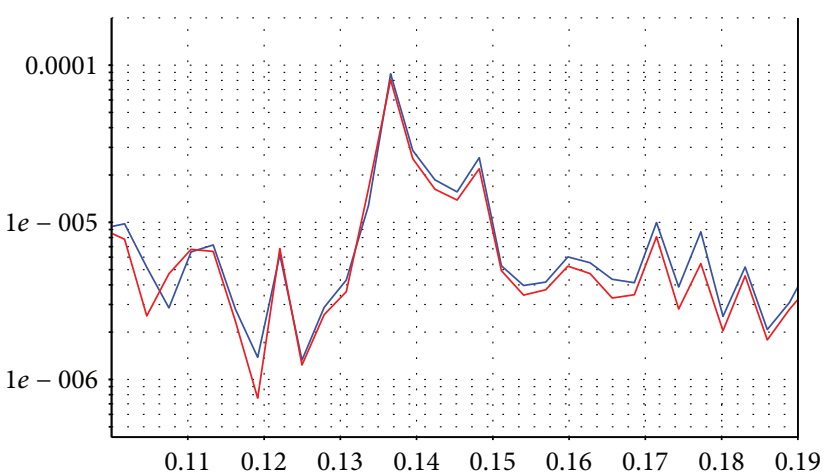

FIGURE 12: Spectrum of the MEP signal before (blue) and after (red) the application of the algorithm with even rotation at a frequency of $0.137 \mathrm{~Hz}$ (blue is before correction and red is after correction).

tp is the operation of calculation of fast Fourier transformation for time record.

The result of the practical application of this algorithm in an experiment is shown in Figure 12.

The contribution of the component conditioned by the unevenness of the platform rotation and the corresponding extra-axial sensitivity of the molecular-electronic sensor was about $10-12 \%$ of the total signal amplitude.

Thus, the general algorithm for control of the analyzed measurement errors in the device for determining the North heading using the dynamic method of the azimuth determination consists in setting the sensitivity axis of the molecular-electronic sensor perpendicular to the platform angular velocity vector, basing on the readings of a sensor measuring the platform rotational speed in time. The errors that remain after this procedure are taken into account by 
subtracting the corresponding parasitic signal from the signal of the molecular-electronic angular motion sensor. Then, the measurements are carried out with the account of the algorithm of the sensor sensitivity to linear acceleration, the North heading is calculated by (11) and (17), and the difference of which is that the term $U_{\text {enc }}(\tilde{\omega}) * k$ is subtracted from the signal of the molecular-electronic sensor $U_{\mathrm{MET}}(\tilde{\omega})$ at the platform rotation frequency.

\section{Conclusion}

Two significant mechanisms of system errors for a dynamic scheme of azimuth determination with the base of MET angular motion sensor have been studied. A theoretical model of MET g-sensitivity error factor has been developed, and an appropriate compensate algorithm was calculated. There have been experimentally studied system errors due to tiny nonoptionality of sensor sensitive axis and the main system axis with the presence of system axis angular motion speed fluctuations (parasite angular acceleration). An appropriate compensation algorithm was developed and approved.

\section{Conflicts of Interest}

The authors declare that there is no conflict of interests regarding the publication of this paper.

\section{Acknowledgments}

This work was supported by the Russian Ministry of Education and Science state assignment under Grant 3.3197.2017/ПЧ.

\section{References}

[1] M. Ilyas, K. Cho, S. H. Baeg, and S. Park, "Drift reduction in pedestrian navigation system by exploiting motion constraints and magnetic field," Sensors, vol. 16, no. 9, 2016.

[2] X. Wang, M. Wu, and J. Feng, "Research of algorithms for fast solving integer ambiguity and dual-GPS heading system," in 2008 International Workshop on Education Technology and Training \& 2008 International Workshop on Geoscience and Remote Sensing, pp. 215-218, Shanghai, China, 2008.

[3] Y. Li, W. Wu, Q. Jiang, and J. Wang, "A new continuous rotation IMU alignment algorithm based on stochastic modeling for cost effective north-finding applications," Sensors, vol. 16, no. 12, 2016.

[4] J. Reiner and M. Naroditsky, "Method and apparatus for determining the geographical heading of a body," US Patent 6502055 B1, G06F 15/00.

[5] M. Naroditsky, "Inertial north finder," US Patent 20070095124 A1.

[6] D. A. Karnick and T. J. Hanson, "Gyroscope north seeker system and method," US Patent 7412775 B1.

[7] E. A. Anikin, E. V. Egorov, and V. M. Agafonov, "Dependence of Self-Noise of the Angular Motion Sensor Based on the Technology of Molecular-Electronic Transfer, on the Area of the Electrodes," IEEE Sensors Letters, vol. 2, no. 2, pp. 1-4, 2018.
[8] I. V. Egorov, A. S. Shabalina, and V. M. Agafonov, "Design and self-noise of MET closed-loop seismic accelerometers," IEEE Sensors Journal, vol. 17, no. 7, pp. 2008-2014, 2017.

[9] H. Huang, B. Carande, R. Tang et al., "A micro seismometer based on molecular electronic transducer technology for planetary exploration," Applied Physics Letters, vol. 102, no. 19, article 193512, 2013.

[10] H. Huang, B. Carande, R. Tang et al., "Development of a micro seismometer based on molecular electronic transducer technology for planetary exploration," in 26th IEEE International Conference on Micro Electro Mechanical Systems (MEMS 2013), pp. 629-632, Taipei, Taiwan, 2013.

[11] K. V. Alekseevich, A. V. Mikhajlovich, S. M. Vladimirovich, and Z. D. Leonidovich, "Method of making electrode assembly of molecular-electronic device for measuring linear and angular motion (versions)," RUS Patent 2394246, 10.07.2010, Bull 19, http://www.freepatent.ru/images/patents/68/2394246/ patent-2394246.pdf.

[12] D. L. Zaitsev, V. M. Agafonov, E. V. Egorov, A. N. Antonov, and V. G. Krishtop, "Precession azimuth sensing with low-noise molecular electronics angular sensors," Journal of Sensors, vol. 2016, Article ID 6148019, 8 pages, 2016.

[13] D. Zaitsev, A. Antonov, and V. Krishtop, "Angular MET sensor for precise azimuth determination," in International Conference on Micro- and Nano-Electronics 2016, Zvenigorod, Russia, 2016, http://proceedings.spiedigitallibrary.org/proceeding.aspx? articleid $=2596130$.

[14] V. M. Agafonov, E. V. Egorov, and D. L. Zaitsev, "Molecular electronic linear accelerometers. Preliminary test results," Gyroscopy and Navigation, vol. 1, no. 4, pp. 246-251, 2010.

[15] D. L. Zaitsev, P. V. Dudkin, T. V. Krishtop et al., "Experimental studies of temperature dependence of transfer function of molecular electronic transducers at high frequencies," IEEE Sensors Journal, vol. 16, no. 22, pp. 7864-7869, 2016.

[16] V. G. Krishtop, "Experimental modeling of the temperature dependence of the transfer function of rotational motion sensors based on electrochemical transducers," Russian Journal of Electrochemistry, vol. 50, no. 4, pp. 350-354, 2014.

[17] V. Agafonov and E. Egorov, "Influence of the electrical field on the vibrating signal conversion in electrochemical (MET) motion sensor," International Journal of Electrochemical Science, vol. 11, pp. 2205-2218, 2016.

[18] E. Egorov, V. Agafonov, S. Avdyukhina, and S. Borisov, "Angular molecular-electronic sensor with negative magnetohydrodynamic feedback," Sensors, vol. 18, no. 1, p. 245, 2018.

[19] D. Zaitsev, V. Agafonov, E. Egorov, A. Antonov, and A. Shabalina, "Molecular electronic angular motion transducer broad band self-noise," Sensors, vol. 15, no. 11, pp. 2937829392, 2015.

[20] E. V. Egorov, I. V. Egorov, and V. M. Agafonov, "Self-noise of the MET angular motion seismic sensors," Journal of Sensors, vol. 2015, Article ID 512645, 5 pages, 2015. 


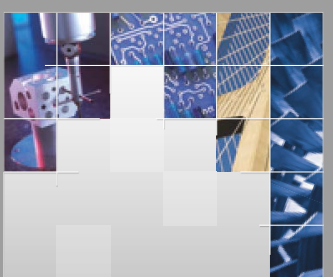

\section{Enfincering}
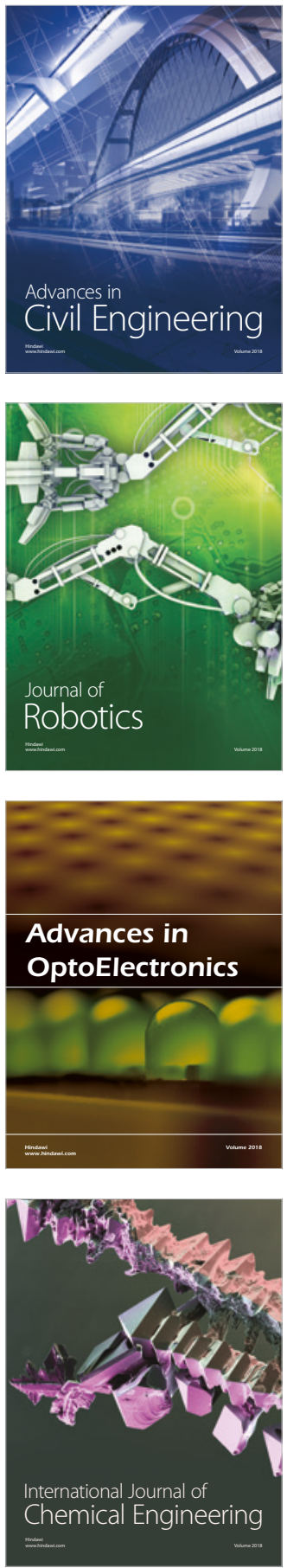

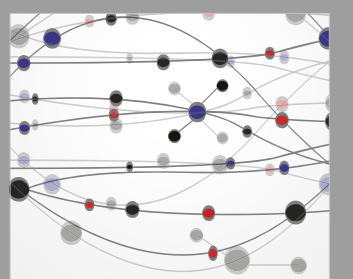

\section{Rotating \\ Machinery}

The Scientific World Journal

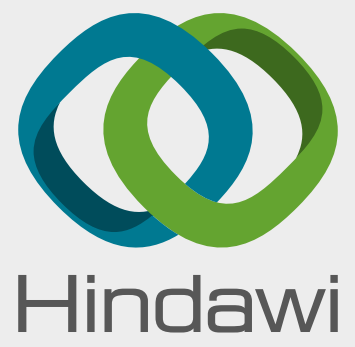

Submit your manuscripts at

www.hindawi.com
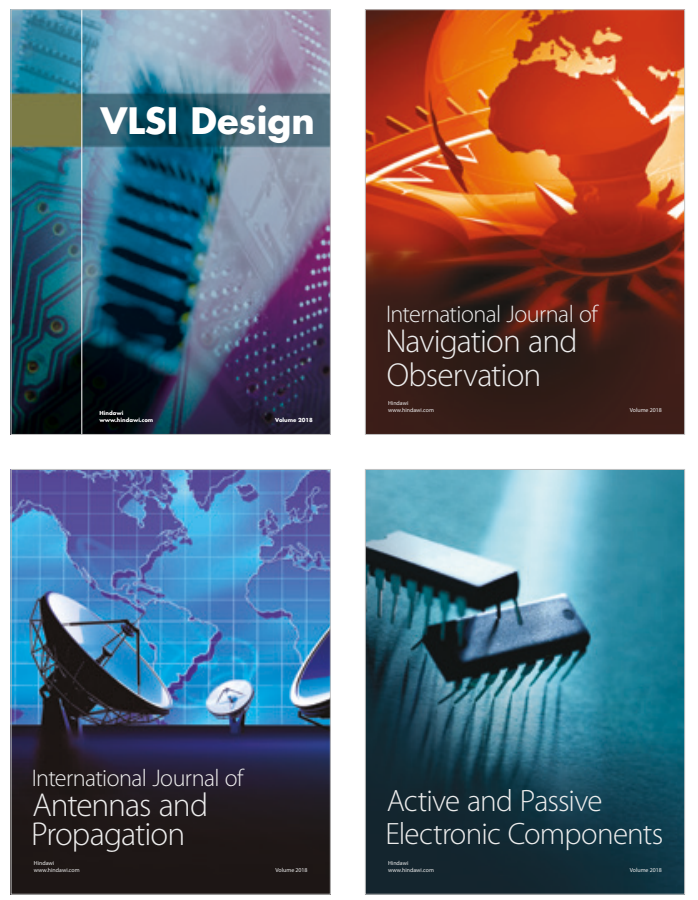
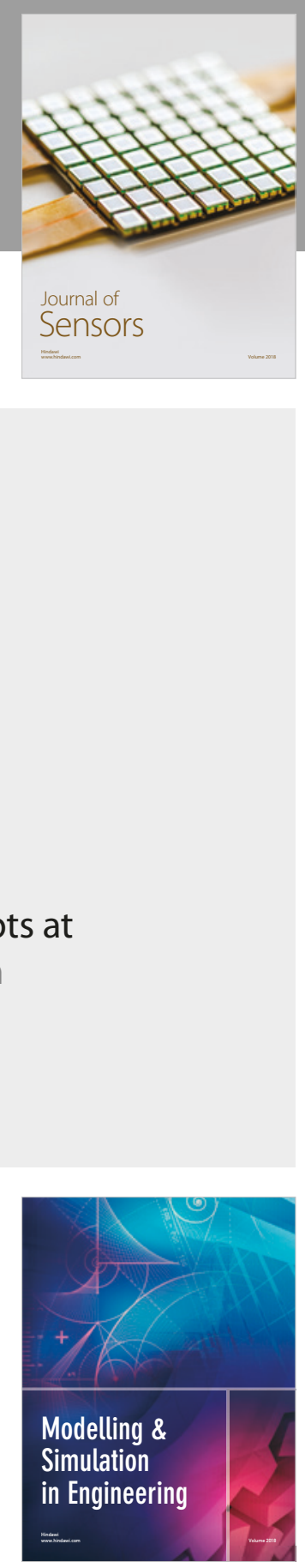

\section{Advances \\ Multimedia}
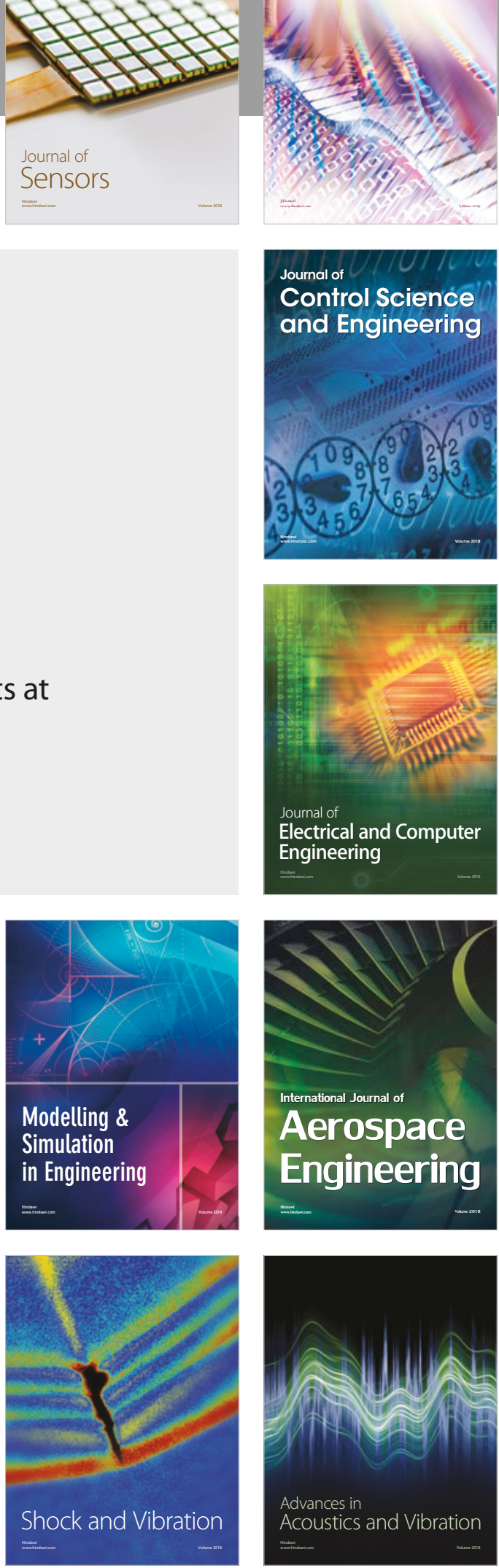